\title{
Colorado Tick Fever Virus
}

National Cancer Institute

\section{Source}

National Cancer Institute. Colorado Tick Fever Virus. NCI Thesaurus. Code C112240.

A species of non-enveloped, spherical viruses with an icosahedral symmetry of triple capsid structure in the Reoviridae family and Coltivirus genus. The genome is composed of segmented, double-stranded RNA. Colorado tick fever viruses are found in adult wood ticks, humans and mammals, and cause Colorado tick fever in humans. Colorado tick fever viruses are transmitted mainly via the bite of infected Rocky Mountain wood ticks (Dermacentor andersoni). 